\title{
A Study on the Effect of Wearing Masks on Stress Response
}

\author{
Zhixing Tian1, Bong-Young Kim2 and Myung-Jin Bae3* \\ ${ }^{1}$ Soong-sil University, Department of Information and telecommunication Engineering, Seoul, 06978, Korea. \\ Orcid Id : 0000-0003-3882-2459 \\ ${ }^{2}$ Soong-sil University, Department of Information and telecommunication Engineering, Seoul, 06978, Korea. \\ Orcid Id : 0000-0002-3553-039X \\ ${ }^{3}$ Soong-sil University, Department of Information and telecommunication Engineering, Seoul, 06978, Korea. \\ Orcid Id : 0000-0002-7585-0400
}

\begin{abstract}
:
Recently, masks have been used more and more frequently. This is not only related to urban air pollution, but also involves the recent global outbreak of new coronaviruses. People are lining up to buy masks in many countries and cities, and the demand for masks in the global market has soared. Due to the global panic caused by the epidemic, people will choose to wear masks to protect themselves against the virus whether they need them or not. The use of masks has become more routine and it has taken longer to wear them. Incorrect use of masks may not provide good protection, also may damage our health. Wearing the mask for a long time can cause poor breathing and even hypoxia. As We knows, the damage to body any organs caused by hypoxia is irreversible, and it also causes psychological disorders. The purpose of this thesis is to explore the psychological impact of wearing a mask on humans by using the correlation between HRV and psychological stress. Research shows that wearing a mask can increase psychological stress. Such research could give people the correct understanding about wearing masks. Thus, people will give priority to the need before wearing a mask, and them will pay more attention to the precautions when wearing a mask. Only using masks reasonably and correctly can protect our health.
\end{abstract}

Keyword: Mask, Air Pollution, COVID19, HRV, SDNN, Stress.

\section{INTRODUCTION}

In the past, masks rarely appeared in our daily lives, and mostly used in special working environments such as hospitals and coal mine sites. However, in recent years, due to air pollution and haze problems, masks have appeared more frequently in our lives. In particular, COVID-19 has spread around the world recently, and the special protective effect of masks makes more people use it, and the wearing time is longer than usual. People are becoming more dependent on masks. But, Wearing a mask with poor ventilation for long time a cause poor breathing and even lack of oxygen. Therefore, excessive use of masks can cause side effects. For example, hypoxia may affect human's boy stress response and increase people's stress.

Increased stress is not a good thing. Greater stress can damage our mental and physical health. It is mainly reflected in the prone to produce negative emotions such as anger, anxiety, depression, which will affect person's normal life and reduce work efficiency. It is important to control stress and keep emotions calm. The purpose of this paper is to find out the impact of wearing masks on human stress response, to arouse people's attention to the problem of excessive use of masks, so that help people use it more reasonably.

In this paper, we use the correlation between stress and heart rate to find out the effect of wearing a mask on Psychological stress. In Chapter 2, we explained the reasons about wearing a mask affects the psychological stress response. In Chapter 3, we explained the relationship between heart rate and stress and the method of analyzing the magnitude of stress with HRV. In Chapter 4, the heart rate was measured with and without a mask, and their respective SDNN values were calculated to obtain the experimental results. Finally, In Chapter 5, conclusions.

\section{CAUSES OF WEARING A MASK AFFECTING STRESS RESPONSE}

KF94 masks (Korean standard) and N95 masks (US standard) are common on the market. KF94 masks are equivalent to P2 filtration and filter out $94 \%$ of particulate matter. It can filter particles above $0.4 \mu \mathrm{m}$. N95 is designed to filter out at least $95 \%$ of the dust and mold in the air. It can filter particles above $0.3 \mu \mathrm{m}$. They can block PM2.5 $(<=2.5 \mu \mathrm{m})$ and virus-borne droplets $(>5 \mu \mathrm{m})$ [1]. This is a reason that it is used by more people. However, they are so tightly sealed that prolonged use or exercise can easily cause brain hypoxia, dizziness, chest tightness and other symptoms. For example, N95 masks are dust masks for industrial use. According to German labor insurance regulations, workers should not wear N95 masks for more than half an hour at a time. Therefore, they are not suitable 
for long-term wear, especially for patients with cardiovascular disease, the elderly, and children [2]. The standard oxygen concentration in the atmosphere is $21 \%$. First, when we put on a mask, a closed space is formed between the mask and the human face. Therefore, when a person inhales again, only after this part of the exhaust gas is inhaled into the human body can the external filtered air be taken in. As a result, the oxygen concentration will reduce in the inhaled air. First, low oxygen concentrations will cause the cells to be hypoxic cause physiological stress. Table 1 is about the adverse reactions of the body when the oxygen content in the air is insufficient [3].

Second, because of the filtering effect and limited transmittance of the mask, breathing resistance is greater when wearing a mask, which makes us feel difficult to breathe. As a result, psychological stress caused by this negative external environment.

Table 1. Adverse reactions of the human in a hypoxic environment

\begin{tabular}{|c|c|}
\hline $\begin{array}{c}\text { Oxygen } \\
\text { concentration }\end{array}$ & Effects and symptoms \\
\hline $15 \%-19 \%$ & $\begin{array}{l}\text { Decrease the person's ability to work, feel hypoxia, can cause problems in the } \\
\text { body's coronary arteries, lungs, circulatory system, etc. }\end{array}$ \\
\hline $12 \%-15 \%$ & $\begin{array}{l}\text { Breathing and pulse pulsations accelerate, and coordination, feeling, and } \\
\text { judgment are impaired. }\end{array}$ \\
\hline $10 \%-12 \%$ & Difficulty breathing, weak judgment, baskets on lips \\
\hline $8 \%-12 \%$ & $\begin{array}{c}\text { Memory loss, unconsciousness, unconsciousness, pale face, lips turning into } \\
\text { basket, nausea and vomiting }\end{array}$ \\
\hline $6 \%-8 \%$ & $\begin{array}{c}8 \text { minutes: death; } 6 \text { minutes: } 50 \% \text { hope for survival; } 4-5 \text { minutes: recoverable } \\
\text { after treatment }\end{array}$ \\
\hline $4 \%-6 \%$ & Coma, convulsions, cessation of breathing to death within 40 seconds \\
\hline
\end{tabular}

The brain consumes a lot of oxygen in our body. When there is insufficient oxygen in the air, the first organ to respond is the brain. The oxygen supply of each organ cell is redistributed through the regulation of autonomic nerves. Hypoxia can cause psychological effects. Some studies have shown that Hypoxia can cause stress, which can increase anxiety and depression [4]. When oxygen is low, depression and suicide rates increase. So, hypoxia is the root cause of increased stress when wearing a mask.

\section{PRINCIPLE OF HEART RATE MEASUREMENT}

\subsection{Heart Rate and Heart Rhythm}

A contraction of the myocardium completes a pulsation of the heart, that is, a heartbeat. Heart rate is the speed of the heartbeat measured by the number of contractions (beats) of the heart per minute (bpm). We usually measure it with an electrocardiogram and a blood pressure monitor. In an electrocardiogram, one pulse is generated for each heartbeat, and the interval between two pulse peaks represents the interval time between two heartbeats. We always call it the N-N interval. It is shown in Figure 1. Exercise, sleep, stress, and illness can all affect heart rate [5].

Heart rhythm is the rhythm of the heart beating. While heart rhythm is regulated entirely by the sinoatrial node under normal conditions, it means that our heart beats regularly. If it doesn't beat regularly, it means that someone's body is abnormal [6]

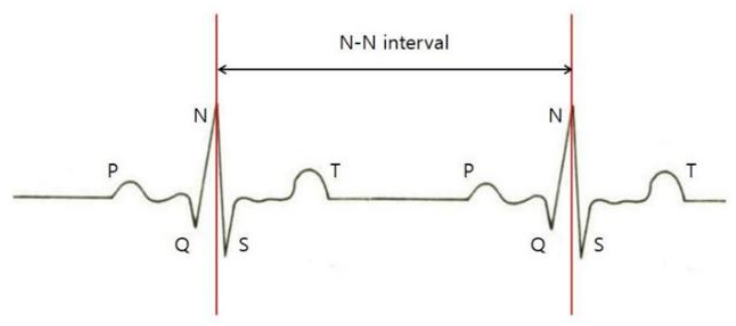

Figure 1. Electrocardiographye (ECG Signal)

\subsection{HRV analysis method}

Sinus arrhythmia is a common change in normal sinus rhythm. Sinus arrhythmias are characterized by irregular rates in which the variation in the $\mathrm{R}-\mathrm{R}$ interval is greater than 0.12 seconds. [7]. The presence of sinus arrhythmias indicates cardiovascular health. This physiological phenomenon of variation in the time interval between heartbeats is defined as Heart rate variability (HRV), and in 1996, the "European Cardiology Society and the North American Society of Pacing and Electrophysiology Special Task Force" proposed a method for analyzing heart rate variability (HRV) And standard guidelines [8]. Many current studies have shown that HRV is related to many physiological 
and psychological phenomena of the human body. HRV is the result of autonomic nervous system activity and balance [9] [10]. Normal physiological activities will maintain a certain HRV change value. When the human body encounters stress, anxiety and other external environmental influences, it will inhibit the variation of heart rhythm and reduce HRV [11]. Figure 2 is a simplified representation of the neurovisceral integration model [12].

There are many methods for HRV analysis. For example, the time domain method is to analyze the standard deviation of the $\mathrm{NN}$ interval. A geometric method is to convert a series of $\mathrm{NN}$ intervals into a geometric pattern. The frequency domain method is to allocate frequency bands and then calculate the number of NN intervals that match each frequency band [8]. The HRV analysis flowchart is shown in Figure 3 [13].

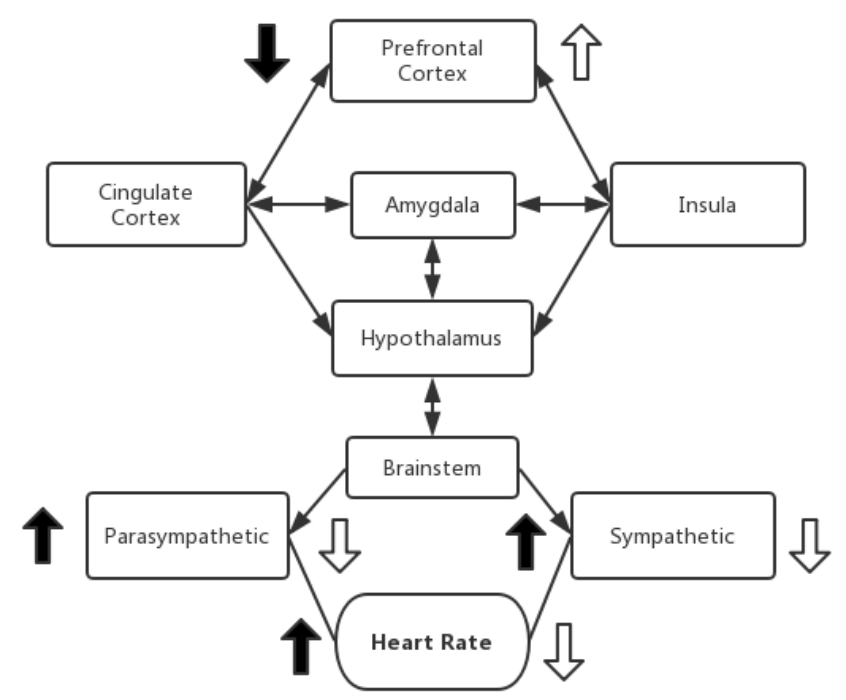

Figure 2. The neurovisceral integration model

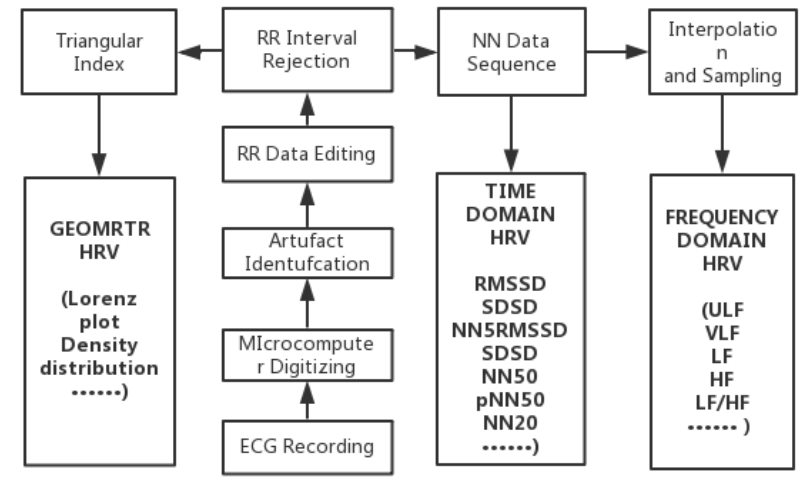

Figure 3. HRV analysis flowchart center

The SDNN analysis used in this paper is to calculate the standard deviation of the NN interval in the time domain. The following is the calculation method of SDNN.

$$
\begin{gathered}
\mathrm{N}-\mathrm{N} \text { Interval }(\mathrm{ms})=\frac{60000}{\text { Heart Rate }(\text { bpm })}, \\
\operatorname{SDNN}(\sigma)=\sqrt{\frac{\sum_{k=1}^{N}\left(x_{k}-\mu\right)^{2}}{N}}
\end{gathered}
$$

where $x_{k}$ is k's N-N Interval, and $\mu$ is N-N Interval's Mean, N is total number of N-N Interval [13]. And the correlation between the SDNN value of HRV analysis method and the magnitude of stress is shown in Table 1 [9].

Table 1. Correlation between SDNN and stress

\begin{tabular}{|c|l|}
\hline Age & SDNN reference \\
\hline & $>50:$ Very good, Autonomic nerve control and stress coping ability \\
& $35 \sim 50:$ Normal, Autonomic nerve control and coping abilities are normal \\
$10 \sim 40$ & $20 \sim 35:$ Low, Stress Disease Risk \& autonomic nerve weakness \\
& $<20:$ Very low, Autonomic nerve high risk of chronic stress related illness \\
\hline $50 \sim 60$ & $>40:$ Very good $20 \sim 30:$ Normal \\
& $15 \sim 20:$ Low \\
\hline
\end{tabular}




\section{EXPERIMENTS AND RESULTS}

Subjects included 20 healthy men and women between the ages of 20 and 45 . In one case, we measured the data for 5 minutes when the Subjects wearing a mask. In another case, we measured the data for 5 minutes when Subjects without a mask. During the measurement, they remained still and calm inside, without interference from other environmental factors. The experimental mask was KF94 protective mask. Then convert the measured NN interval data into SDNN analysis, and finally compare the SDNN value in the two cases, then correspond the SDNN value to the pressure index table. Finally, the experimental results are obtained [14][15][16]. The experimental principle is shown in Figure 4.

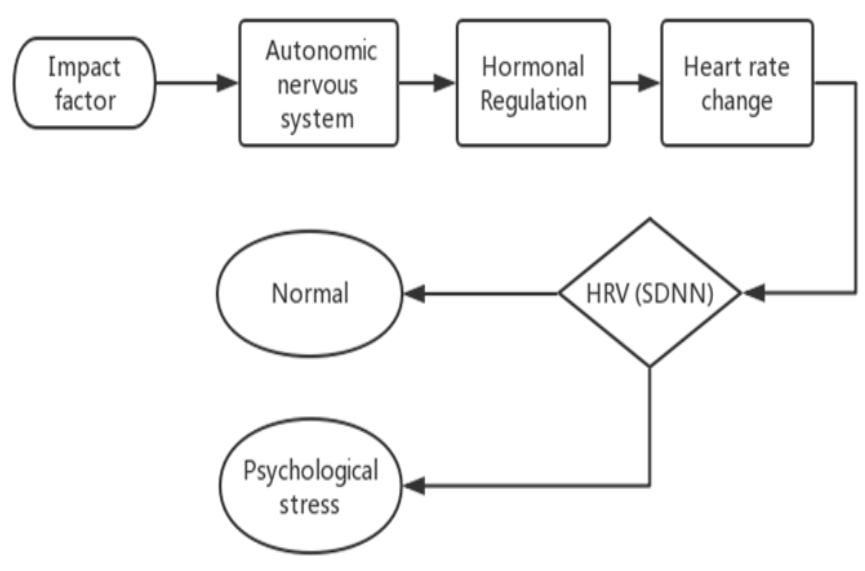

Figure 4. Experimental principle of HRV

Figure 5 is the change of NN interval when person A does not wear a mask. Figure 6 is the change of NN interval when A wears a mask. when not wearing a mask (figure 5), the $\mathrm{NN}$ interval change fluctuates greatly, and the deviation from the average value is large. When wearing a mask, the NN interval changes little and is closer to the average. So, the SDNN is smaller in this case, and the heart rate does not change much, it means that wearing a mask will increase psychological stress.

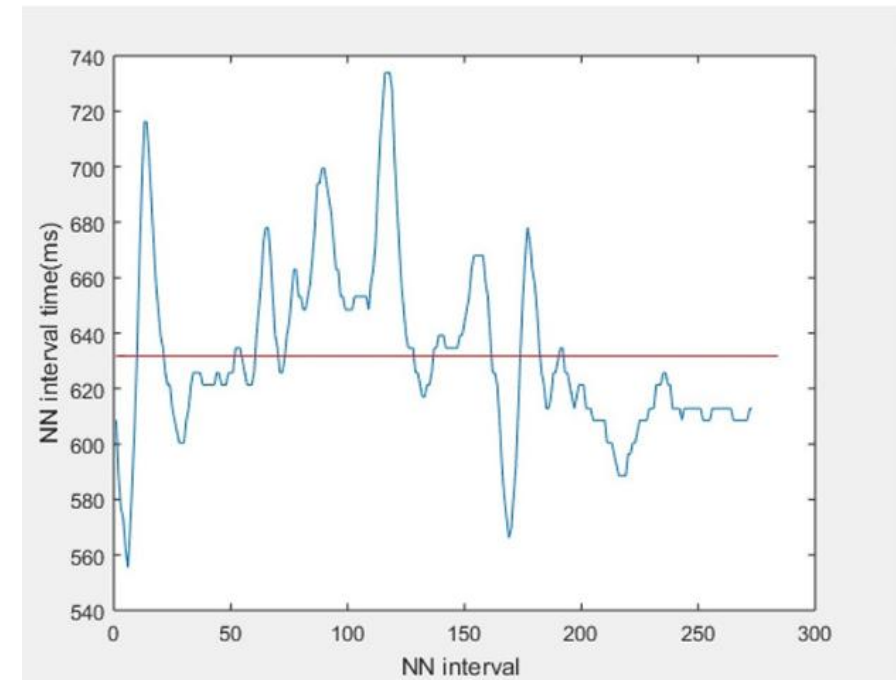

Figure 5. The change of NN interval when people A does not wear a mask

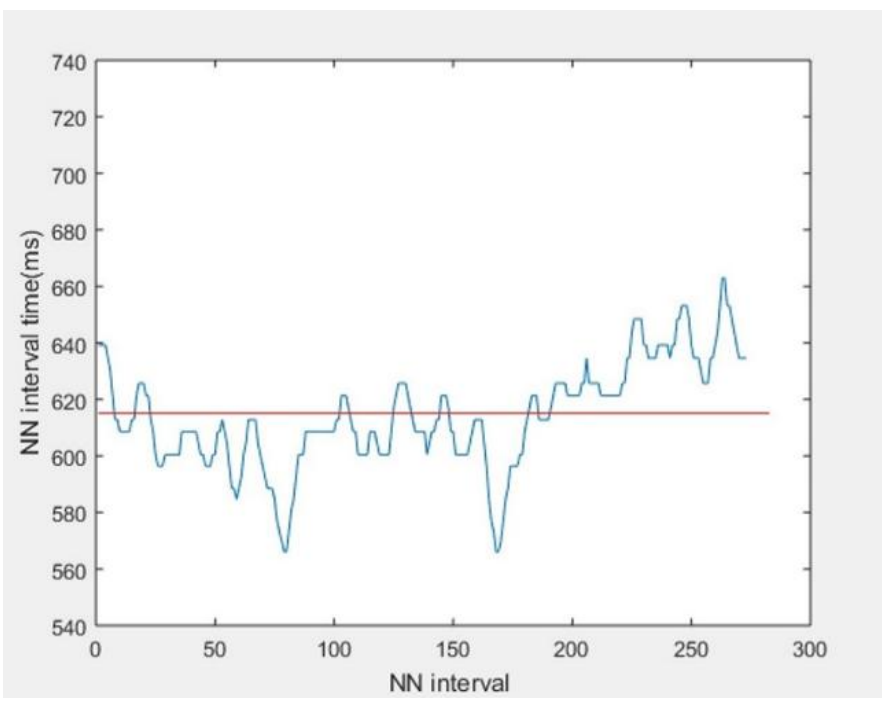

Figure 6. The change of NN interval when people A wears a mask

Table 2 is the average heart rate of 20 subjects who participated in the heart rate test, SDNN experimental results and the difference value $\triangle \mathrm{SDNN}$ with and without a mask. Figure 7 is a statistical line chart of SDNN values of 20 subjects. 
Table 2. Results of Heart Rate measurement

\begin{tabular}{|c|c|c|c|c|c|}
\hline \multirow{2}{*}{} & \multicolumn{2}{|c|}{ No mask } & \multicolumn{2}{c|}{ Mask on } & \\
\cline { 2 - 6 } & $\begin{array}{c}\text { Average heart } \\
\text { rate(bpm) }\end{array}$ & SDNN 1(S1) & $\begin{array}{c}\text { Average heart } \\
\text { rate(bpm) }\end{array}$ & SDNN 2(S2) & $\Delta S D N N$ \\
\hline A & 82 & 39.29 & 87 & 23.53 & $\mathbf{- 1 5 . 7 6}$ \\
\hline B & 93 & 42.72 & 93 & 38.15 & $\mathbf{- 4 . 5 7}$ \\
\hline C & 69 & 38.30 & 64 & 27.92 & $\mathbf{- 1 0 . 3 8}$ \\
\hline D & 87 & 68.70 & 89 & 60.58 & $\mathbf{- 8 . 1 2}$ \\
\hline E & 90 & 40.63 & 92 & 33.59 & $\mathbf{- 7 . 0 4}$ \\
\hline F & 87 & 35.46 & 87 & 36.51 & $\mathbf{1 . 0 5}$ \\
\hline G & 85 & 59.64 & 80 & 48.35 & $\mathbf{- 1 1 . 2 9}$ \\
\hline H & 76 & 39.41 & 80 & 40.02 & $\mathbf{0 . 6 1}$ \\
\hline I & 56 & 23.49 & 50 & 20.38 & $\mathbf{- 3 . 1 1}$ \\
\hline J & 79 & 46.70 & 85 & 42.63 & $\mathbf{- 4 . 0 7}$ \\
\hline K & 80 & 26.80 & 81 & 20.06 & $\mathbf{- 6 . 7 4}$ \\
\hline L & 76 & 65.22 & 78 & 56.86 & $\mathbf{- 8 . 3 6}$ \\
\hline M & 96 & 41.86 & 95 & 39.08 & $\mathbf{- 2 . 7 8}$ \\
\hline N & 88 & 61.89 & 85 & 53.82 & $\mathbf{- 8 . 0 7}$ \\
\hline O & 94 & 40.73 & 91 & 32.82 & $\mathbf{- 7 . 9 1}$ \\
\hline P & 78 & 44.97 & 80 & 33.52 & $\mathbf{- 1 1 . 4 5}$ \\
\hline Q & 69 & 43.70 & 72 & 59.15 & $\mathbf{1 5 . 4 5}$ \\
\hline R & 77 & 56.77 & 80 & 51.79 & $\mathbf{- 4 . 9 8}$ \\
\hline S & 86 & 52.99 & 83 & 44.51 & $\mathbf{- 8 . 4 8}$ \\
\hline T & 95 & 25.14 & 96 & 35.45 & $\mathbf{1 0 . 3 1}$ \\
\hline
\end{tabular}

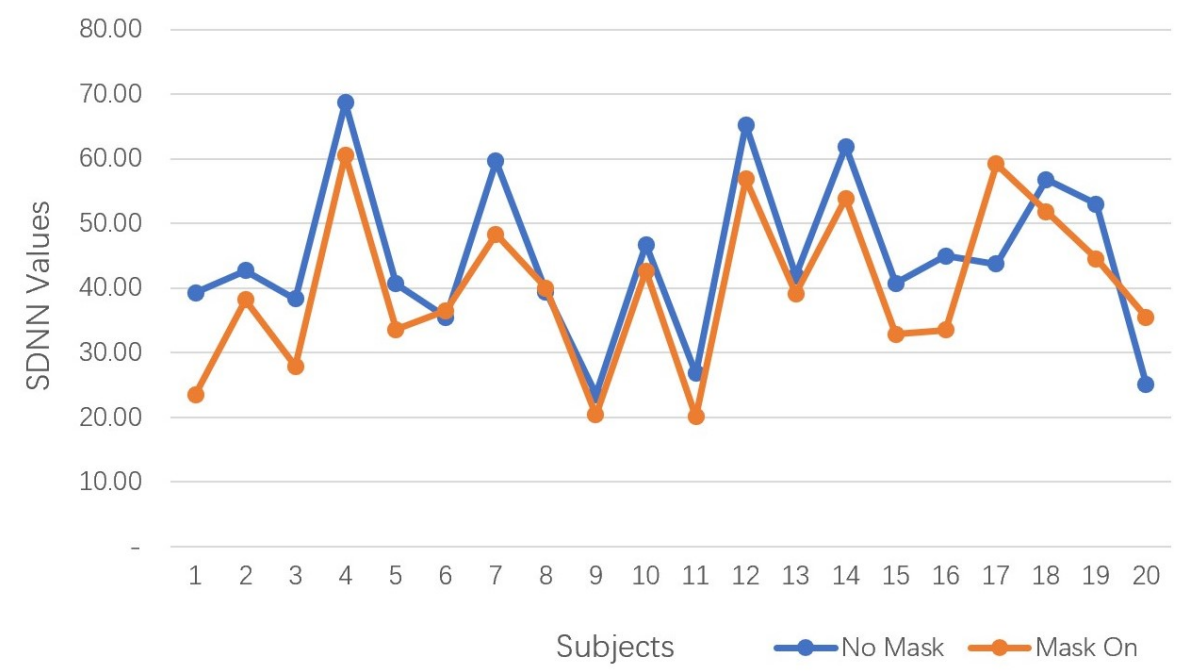

Figure 7. SDNN values of 20 subjects. 
Comparing the experimental results in Table 2 and figure 7, we find that SDNN wearing a mask is different from SDNN without a mask, and 16 of 20 people have reduced the value of SDNN due to wearing a mask. There are 16 people experienced a significant increase in stress index after wearing a mask, accounting for $80 \%$ of the Subjects population, Two people were almost unaffected, accounting for $10 \%$, and two people had reduced stress, accounting for $10 \%$. Therefore, in most cases, wearing a mask will affect human stress response and increase stress index. Because wearing a mask impedes breathing, affects the regulation of our autonomic nervous system, Finally, the changes in heart rate were suppressed [17] [18]. In other words, we will get more stress than without a mask.

\section{CONCLUSION}

It is well known that wearing a mask may cause breathing problems and hypoxia. And this situation will become more serious with the time of wearing the mask and the degree of protection of the mask. For example, N95 masks are dust masks for industrial use. According to German labor insurance regulations, workers should not wear N95 masks for more than half an hour at a time. KF94 is similar to N95. Prolonged wearing may cause permanent damage to the respiratory system. Due to the lack of oxygen in the brain, it also damages our nervous system, causing adverse physiological reactions, such as dizziness, chest tightness, and psychological reactions about anxiety and depression.

In this paper, we use the correlation between HRV and stress to explore whether wearing a mask will cause stress response on the human body. According to the experimental results obtained by HRV's SDNN analysis method, we found that wearing a mask did reduce the SDNN value of $80 \%$ of the test subjects. This is only a test result of wearing a mask for 5 minutes. This is only the result of wearing a mask for 5 minutes. We believe that as the duration of wearing a mask is longer, the more severe the situation of hypoxia, the more severe the body 's response to hypoxia will be. The range of SDNN value decline will be greater. This also means that the people will suffer more stress, which harm people's health.

Therefore, people need to know the negative effects of the mask and use the mask appropriately. It is not recommended to use excessive high protection level and tightness under normal circumstances mask. Also, it is not recommended to use a high protection level mask during exercise. When using N95 and KF94 masks for a long time to prevent the COVID-19 virus, sometimes, it is important to take off the mask in a wellventilated place and breathe in fresh air. As it is inevitable to wear a high protection level mask due to viruses such as COVID-19, it is necessary to protect physical and mental health through proper use of masks.

\section{REFERENCE}

[1] Difference between kf94 mask and n95 mask. https://www.best73.com/news/13332.html.

[2] ifeng.com." Will the N95 mask be hypoxia for a long time?

[http://fashion.ifeng.com/2body/special/2031yy169/]

[3] Oxygen deficiency-confined space. [https://mydaum00.tistory.com/m/300]

[4] Central Daily News. " If you lack oxygen, you'll be happy hormones. $\downarrow$ If you take an oxygen shower, you will feel depressed and anxious. [https://news.joins.com/article/23008248]

[5] "All About Heart Rate (Pulse)". American Heart Association. 22 Aug 2017. Retrieved 25 Jan 2018.

[6] Jönsson P (January 2007). "Respiratory sinus arrhythmia as a function of state anxiety in healthy individuals". International Journal of Psychophysiology. 63 (1): 48-54.

[7] Soos, Michael P.; McComb, David (2019-03-09). "Sinus Arrhythmia".

[8] CAMM, A. John, et al. "Heart rate variability: standards of measurement, physiological interpretation and clinical use". Task Force of the European Society of Cardiology and the North American Society of Pacing and Electrophysiology. Circulation, Vol.93, No.5, pp.10431065, 1996.

[9] Bong-Young Kim, Myung-Jin Bae."A study on the effect of jogging on stress reduction". International Journal of Engineering Research and Technology 11(9), pp. 13631370.

[10] Bong-Young Kim, Zhixing Tian, Myung-Jin Bae. "A study on the causes of revenge psychology of Klaxon sound". International Journal of Engineering Research and Technology Volume 12, Issue 12, 2019, Pages 2947 2952.

[11] Zhixing Tian, Bong-Young Kim, Myung-Jin Bae. "A study on the Improvement of Klaxon Sound". International Journal of Engineering Research and Technology12(12), pp. 2947-2952.

[12] Nikolin S, Boonstra TW, Loo CK, Martin D (2017-0803). "Combined effect of prefrontal transcranial direct current stimulation and a working memory task on heart rate variability". PLOS ONE. 12 (8): e0181833.

[13] Bong-Young Kim, Myung-Jin Bae. "A study on the stress reduction effect of reading aloud the book using HRV". International Journal of Engineering Research and Technology Volume 12, Issue 9, 2019, Pages 1457-1461.

[14] Appelhans, Bradley M.; Luecken, Linda J. (September 2006). "Heart Rate Variability as an Index of Regulated Emotional Responding". Review of General Psychology. 10 (3): 229-240.

[15] Heart Rate Variability Analysis System / Clinical Information, Medicore, Korea. 
International Journal of Engineering Research and Technology. ISSN 0974-3154 Vol.13, No.4 (2020), pp. 807-813

(C) International Research Publication House. https://dx.doi.org/10.37624/IJERT/13.4.2020.807-813

[16] Koolhaas J, et al. (2011). "Stress revisited: A critical evaluation of the stress concept". Neuroscience and Biobehavioral Reviews. 35 (5): 1291-1301.

[17] McKlveen, Jessica M.; Myers, Brent; Herman, James P. "The Medial Prefrontal Cortex: Coordinator of Autonomic, Neuroendocrine, and Behavioral Responses to Stress". Journal of Neuroendocrinology. 27 (6): 446456
[18] Winbo, A., Paterson, D.J. " The Brain-Heart Connection in Sympathetically Triggered Inherited Arrhythmia Syndromes ". Heart Lung and Circulation Volume 29, Issue 4, April 2020, Pages 529-537

[19] Tohoku News Website. "The right way to wear a mask". [http://liaoning.nen.com.cn/system/2020/03/19/0209933 13.shtml] 